\title{
Molecular evolution of glutamine synthetase II: Phylogenetic evidence of a non-endosymbiotic gene transfer event early in plant evolution
}

\author{
Sohini Ghoshroy*1, Manfred Binder ${ }^{1}$, Aurélien Tartar² and Deborah L Robertson ${ }^{1}$
}

\begin{abstract}
Background: Glutamine synthetase (GS) is essential for ammonium assimilation and the biosynthesis of glutamine. The three GS gene families (GSI, GSII, and GSIII) are represented in both prokaryotic and eukaryotic organisms. In this study, we examined the evolutionary relationship of GSII from eubacterial and eukaryotic lineages and present robust phylogenetic evidence that GSII was transferred from $\gamma$-Proteobacteria (Eubacteria) to the Chloroplastida.

Results: GSII sequences were isolated from four species of green algae (Trebouxiophyceae), and additional green algal (Chlorophyceae and Prasinophytae) and streptophyte (Charales, Desmidiales, Bryophyta, Marchantiophyta,

Lycopodiophyta and Tracheophyta) sequences were obtained from public databases. In Bayesian and maximum likelihood analyses, eubacterial $\left(G S \|_{B}\right)$ and eukaryotic $\left(G S \|_{E}\right)$ GSII sequences formed distinct clades. Both $G S \|_{B}$ and GSII

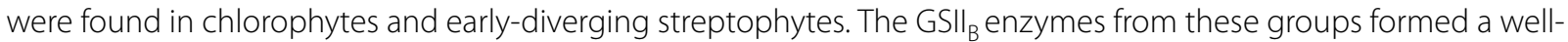
supported sister clade with the $\gamma$-Proteobacteria, providing evidence that GSII in the Chloroplastida arose by horizontal gene transfer (HGT). Bayesian relaxed molecular clock analyses suggest that GSII ${ }_{B}$ and $G S \|_{E}$ coexisted for an extended period of time but it is unclear whether the proposed HGT happened prior to or after the divergence of the primary endosymbiotic lineages (the Archaeplastida). However, GSII genes have not been identified in glaucophytes or red algae, favoring the hypothesis that $G S \|_{B}$ was gained after the divergence of the primary endosymbiotic lineages. Duplicate copies of the GSII ${ }_{B}$ gene were present in Chlamydomonas reinhardtii, Volvox carterif. nagariensis, and Physcomitrella patens. Both GSII $\|_{B}$ proteins in C. reinhardtii and V. carterif. nagariensis had N-terminal transit sequences, indicating they are targeted to the chloroplast or mitochondrion. In contrast, GSII ${ }_{B}$ proteins of P. patens lacked transit sequences, suggesting a cytosolic function. GSII ${ }_{B}$ sequences were absent in vascular plants where the duplication of GSII replaced the function of GSII .
\end{abstract}

Conclusions: Phylogenetic evidence suggests GSII in Chloroplastida evolved by HGT, possibly after the divergence of the primary endosymbiotic lineages. Thus while multiple GS isoenzymes are common among members of the Chloroplastida, the isoenzymes may have evolved via different evolutionary processes. The acquisition of essential enzymes by HGT may provide rapid changes in biochemical capacity and therefore be favored by natural selection.

\section{Background}

Glutamine synthetase (GS: E.C. 6.3.1.2) catalyzes the ATP-dependent formation of Gln from Glu and $\mathrm{NH}_{4}{ }^{+}$ and is considered one of the oldest functioning enzymes $[1,2]$. The GS gene superfamily includes three distinct

* Correspondence: sghoshroy@clarku.edu

1 Clark University, Biology Department, 950, Main Street, Worcester, MA 01610, USA

Full list of author information is available at the end of the article classes, GSI, GSII and GSIII, each differing in molecular size and number of subunits in the holoenzyme [3,4]. The distribution of the three classes is variable within the three domains of life and instances of multiple GS isoenzymes from different families functioning in the same organism are not uncommon in both eubacteria and eukaryotes [3,5-8]. These observations suggest the gene families arose early and prior to the divergence of the prokaryotes and eukaryotes [9-11]. 
The evolutionary history of the GS superfamily is complicated and gene transfer events are one among many forces that have shaped the history of these genes. Among prokaryotes, there is phylogenetic evidence of horizontal gene transfer (HGT) of GSI genes between members of the Archaea and Eubacteria [9]. Evidence for secondary endosymbiotic gene transfer of GSII from the red algal endosymbiont to the nucleus of the heterokont host has also been presented [11]. Members of the GSII gene family are found in both eubacterial and eukaryotic lineages. The identification of GSII genes in the plant symbiont Bradyrhizobium japonicum lead Carlson and Chelm [12] to hypothesize that the gene evolved via HGT from vascular plants to bacteria. However, this hypothesis was not supported by subsequent phylogenetic analyses $[11,13]$, which established distinct eukaryotic $\left(\mathrm{GSII}_{\mathrm{E}}\right)$ and eubacterial $\left(\mathrm{GSII}_{\mathrm{B}}\right)$ clades.

The supergroup Archaeplastida [14], consisting of Glaucophyta, Rhodophyceae and Chloroplastida, harbors members of GSII gene family that are well characterized in vascular plants but not in other lineages within the supergroup. In general, vascular plants express multiple GS isoenzymes that are localized to either cytosol or chloroplast. The isoenzymes are nuclear encoded, and in most angiosperms a single nuclear gene encodes the chloroplast isoenzyme, while a small nuclear gene family encodes multiple cytosolic isoenzymes that are expressed in tissue-specific and developmentally-regulated patterns [15-18]. Previous phylogenetic analyses of chloroplast and cytosolic isoenzymes support the hypothesis that the isoenzymes in angiosperms evolved via a gene duplication event that preceded the divergence of monocots and dicots $[19,20]$.

Biochemical studies of green algae provided the first evidence that, as observed in vascular plants, multiple GSII isoenzymes are expressed and localized to the cytosol and chloroplasts within these organisms [21,22]. Phylogenetic analyses incorporating the two GSII isoforms characterized in Chlamydomonas reinhardtii [23] uncovered an unusual disparity between the two enzymes [20]. The cytosolic GSII sequence clustered with the vascular plants while the plastid sequence branched more basally and appeared to associate with the eubacterial sequences.

Here we examined the evolutionary relationship of the GSII gene family and use increased taxonomic sampling in Chloroplastida to determine if the basally branching, eubacterial-like GSII $_{B}$ was broadly distributed. GSII sequences were obtained from four members of the Trebouxiophyceae (Chlorophyta) by PCR amplification using degenerate and gene specific primers. Additional GSII sequences for members of the green algae (Chlorophyta and Prasinophytae) and streptophytes (Mesostigma, Charales, Desmidiales, Bryophyta, March- antiophyta, Lycopodiophyta and Tracheophyta) were obtained from publicly available databases, including genome and EST projects. We also increased taxonomic sampling within Eubacteria (to date, GSII genes have not been reported from Archaea). $\mathrm{GSII}_{\mathrm{E}}$ and $\mathrm{GSII}_{\mathrm{B}}$ sequences were identified in members of the green algae and earlydiverging streptophytes. Phylogenetic analyses provide support for the hypothesis that $\mathrm{GSII}_{\mathrm{B}}$ was gained in the Chloroplastida from the Eubacteria via a HGT event after the divergence of primary photosynthetic groups.

\section{Results and Discussion \\ Amplification of GSII genes}

Complete GSII mRNA sequences were obtained from Pseudochlorella sp. CCAP211/1A, Chlorella luteoviridis, Auxenochlorella protothecoides, and Prototheca zopfii. A GSII sequence was also obtained for Pseudochlorella sp. CCAP211/1A that included $912 \mathrm{bp}$ of the ORF and all of the 3'UTR. GenBank accession numbers and characteristics of the transcripts obtained in this study are summarized in Table 1.

\section{Eukaryotic GSII phylogeny}

Phylogenetic analyses of GSII amino acid sequences resulted in a well-resolved tree. Assuming the root of the tree lies outside the major eukaryotic clade, there was a clear separation of the eukaryotic $\left(\mathrm{GSII}_{\mathrm{E}}\right)$ and eubacterial $\left(\mathrm{GSII}_{\mathrm{B}}\right)$ enzymes (Figure 1). Within the eukaryote clade, the opisthokonts (fungi+animals) and photosynthetic eukaryotes formed separate groups (Figures 1 and 2). The $\mathrm{GSII}_{\mathrm{E}}$ proteins from streptophytes, chlorophytes, rhodophytes, and chromalveolates formed distinct clades, each with strong to moderate support. The position of the heterokont sequences within this clade is consistent with previous analyses that provide evidence that $\mathrm{GSII}_{\mathrm{E}}$ in heterokonts arose via endosymbiotic gene transfer [11]. Sequences from Chlorophyta (green algae, including representatives of the Chlorophyceae and Trebouxiophyceae) diverged from a basal node within the photosynthetic clade and the Chloroplastida (eukaryotes with chlorophylls $a$ and $b$ ) were not monophyletic. However, the deeper nodes within the photosynthetic eukaryotic clade were not well supported and thus the branching pattern within the clade is unresolved (Figure 2). The streptophyte $\mathrm{GSII}_{\mathrm{E}}$ sequences formed two major groupings; one group contained protein sequences targeted to the chloroplast of angiosperms and the other contained protein sequences of non-vascular and vascular plants that are targeted to the cytosol. Multiple $\mathrm{GSII}_{\mathrm{E}}$ genes were also observed in the gymnosperms (Рinus spp.) but to date, these appear to function in the cytosol and evidence of plastid targeted isoenzymes is lacking [24]. 
Table 1: Summary of the GSII sequences characterized in the present study

\begin{tabular}{|c|c|c|c|c|c|c|c|}
\hline \multirow[t]{2}{*}{ Taxa } & \multicolumn{4}{|c|}{ Sequences Obtained } & \multicolumn{3}{|c|}{$\%$ GC content } \\
\hline & Accession Number & Length (bp) & ORF (bp) & Amino Acids & ORF & 5' UTR & 3' UTR \\
\hline \multicolumn{8}{|l|}{ GSII $_{\mathrm{E}}$ Sequences } \\
\hline Pseudochlorella sp. CCAP211/1A (2) & GQ465769 & 1486 & 1137 & 378 & 63.32 & 53.19 & 55.12 \\
\hline Chlorella luteoviridis UTEX 28 & GQ465770 & 1675 & 1146 & 381 & 56.20 & 46.27 & 46.17 \\
\hline Auxenochlorella protothecoides & GQ465771 & 1621 & 1161 & 386 & 67.96 & 58.18 & 67.28 \\
\hline Prototheca zopfii ATCC16527 & GQ465772 & 1632 & 1158 & 385 & 69.26 & 71.54 & 72.42 \\
\hline \multicolumn{8}{|l|}{ GSII $_{B}$ Sequences } \\
\hline Pseudochlorella sp. CCAP211/1A (1) & GQ491030 & 1266 & 912 & 303 & 56.47 & n.d. & 47.93 \\
\hline
\end{tabular}

\section{Evidence for the HGT of GSII $_{B}$}

The GSII $_{B}$ clade comprised sequences from eubacteria and some members of the Chloroplastida (green algae, liverworts, and mosses; Figures 1 and 3). The Chloroplastida sequences formed a single clade nested within the eubacterial sequences and branching within the clade was similar to predicted organismal phylogenies [25].

$\mathrm{GSII}_{\mathrm{B}}$ sequences are not broadly represented among eubacteria but were identified in members of the Bacteriodetes/Flavobacteria/Cytophaga, Planctomycetes, Verrucomicrobia, Actinobacteria, and the $\alpha-$ and $\gamma$ Proteobacteria (Figure 3; Additional files 1 and 2). The Chloroplastida $\mathrm{GSII}_{\mathrm{B}}$ was sister to $\gamma$-Proteobacteria with strong (Bayesian posterior probability $=1.0$ ) to moderate support (likelihood bootstrap support $=70 \%$ ). The $\gamma$-Proteobacteria + Chloroplastida GSII $_{B}$ clade was sister to the Actinobacteria, but this association was not strongly supported. The $\alpha$-Proteobacteria GSII $_{B}$ sequences were not closely related to the $\gamma$-Proteobacteria + Chloroplastida $\mathrm{GSII}_{\mathrm{B}}$ clade, which makes the possibility of $\mathrm{GSII}_{\mathrm{B}}$ gain via mitochondrial endosymbiosis unlikely. The $\alpha$-Proteobacteria $\mathrm{GSII}_{\mathrm{B}}$ were nested within the Verrucomicrobia and thus, we cannot exclude the possibility of an HGT event within the $\alpha$-Proteobacteria lineage that obscures the mitochondrial origin of the $\mathrm{GSII}_{\mathrm{B}}$ gene in the Chloroplastida. However, the lack of detection of $\mathrm{GSII}_{\mathrm{B}}$ in genomes of other eukaryotic lineages reduces the likelihood of a mitochondrial origin. In addition, EST and genome analyses of other photosynthetic eukaryotes (Glaucophyta, Rhodophyceae and Chromalveolates) and extant cyanobacteria [26,27], have not uncovered GSII $_{B}$ sequences, reducing the possibility that $\mathrm{GSII}_{B}$ was acquired via plastid endosymbiosis. Thus, we propose that $\mathrm{GSII}_{\mathrm{B}}$ in the Chloroplastida arose via a HGT from $\gamma$ Proteobacteria early in plant evolution.

$\mathrm{GSII}_{\mathrm{B}}$ sequences are not broadly distributed among eubacterial lineages and to date, within $\gamma$-Proteobacteria, only the genera represented in our analyses have annotated $\mathrm{GSII}_{\mathrm{B}}$ sequences deposited in GenBank. Assuming the GS superfamily evolved prior to the divergence of the three domains of life [9-11], the distribution of $\mathrm{GSII}_{B}$ sequences suggests the gene has been lost in several lineages of Eubacteria and the Archaea. The analysis of $\mathrm{GSII}_{\mathrm{B}}$ may become more robust as additional eubacterial $\mathrm{GSII}_{\mathrm{B}}$ become available through genome sequencing projects. However, gene loss may make the identification of the true donor of $\mathrm{GSII}_{\mathrm{B}}$ to the Chloroplastida difficult.

An alternative explanation for the limited distribution of $\mathrm{GSII}_{\mathrm{B}}$ among the eubacteria is that the gene was transferred to the eubacteria from an eukaryotic donor. The possibility of an HGT from Chloroplastida to the $\gamma$-Proteobacteria is not supported by our phylogenetic analyses as it implies that the eubacterial sequences would nest within the $\mathrm{GSII}_{\mathrm{E}}$ clade; which has not been observed in our phylogenetic analyses. Eukaryote to eubacterial HGT might be supported if $\mathrm{GSII}_{\mathrm{B}}$ were found in diverse lineages of eukaryotes. Further investigation of GSII diversity in the eukaryotic lineages not represented in our study (e.g., Rhizaria, Excavata and Amoebozoa) will contribute to our understanding of the distribution and evolution of $\mathrm{GSII}_{\mathrm{B}}$. Given the data at hand, however, the hypothesis that $\mathrm{GSII}_{\mathrm{B}}$ arose in the Chloroplastida via HGT remains the most parsimonious. 


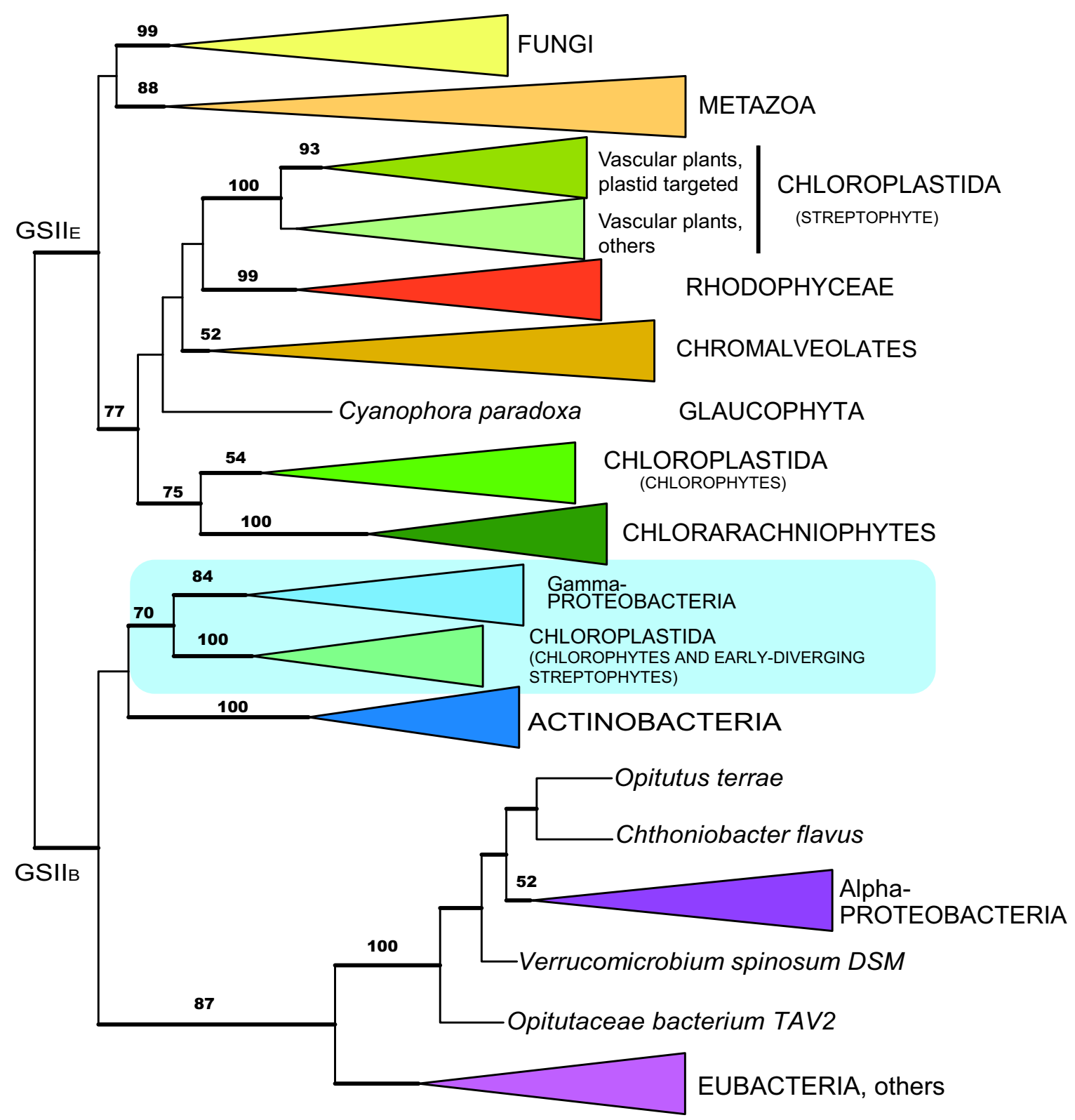

Substitutions /site

Figure 1 Evolutionary relationships among GSII enzymes from prokaryotes and eukaryotes. The phylogenetic analyses were based on 333 amino acid characters from 196 taxa. The 50\% majority-rule consensus tree from the Bayesian analyses [48,49] is shown as inferred from 20,002 trees as described in the Methods. Nodes with BBP support > 0.95 are represented by thick lines. RAxML [50,51] bootstrap values are indicated for nodes recovered in both analyses. RAxML values are not indicated for terminal bifurcations. Eubacterial GSII ${ }_{B}$ were used as the outgroup and considered monophyletic. The area of the triangles representing collapsed clades is not proportional to the number of taxa within the clade. 


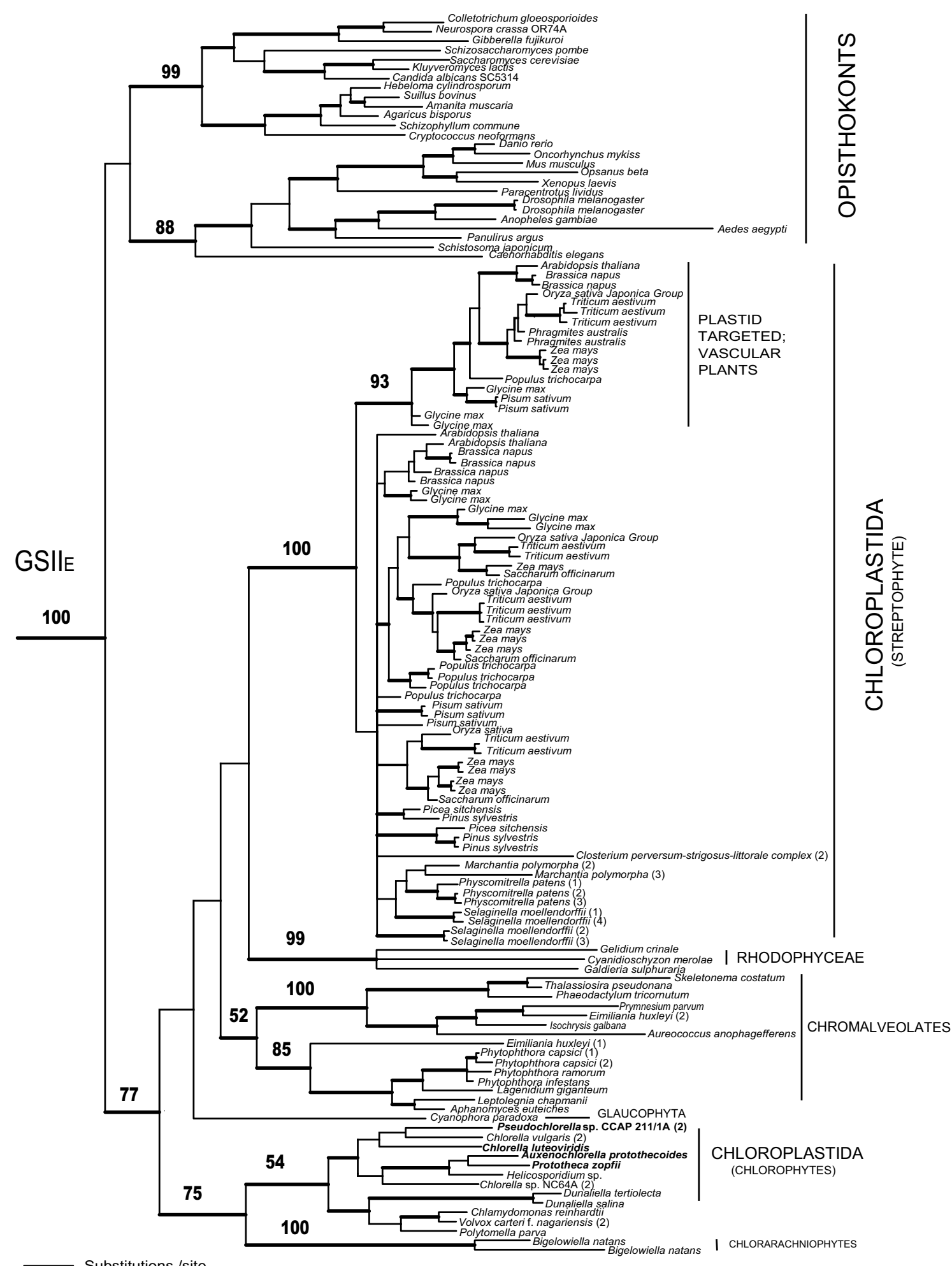

Figure 2 Evolutionary relationship of $\mathrm{GSII}_{\mathrm{E}}$ genes from eukaryotes, terminal taxa expanded. Phylogenetic analyses are as described in figure 1. Nodes with BBP support $>0.95$ are represented by thick lines. RAxML bootstrap values are indicated for major nodes. RAxML values are not indicated for terminal bifurcations. Sequences characterized in the present study are shown in bold. 


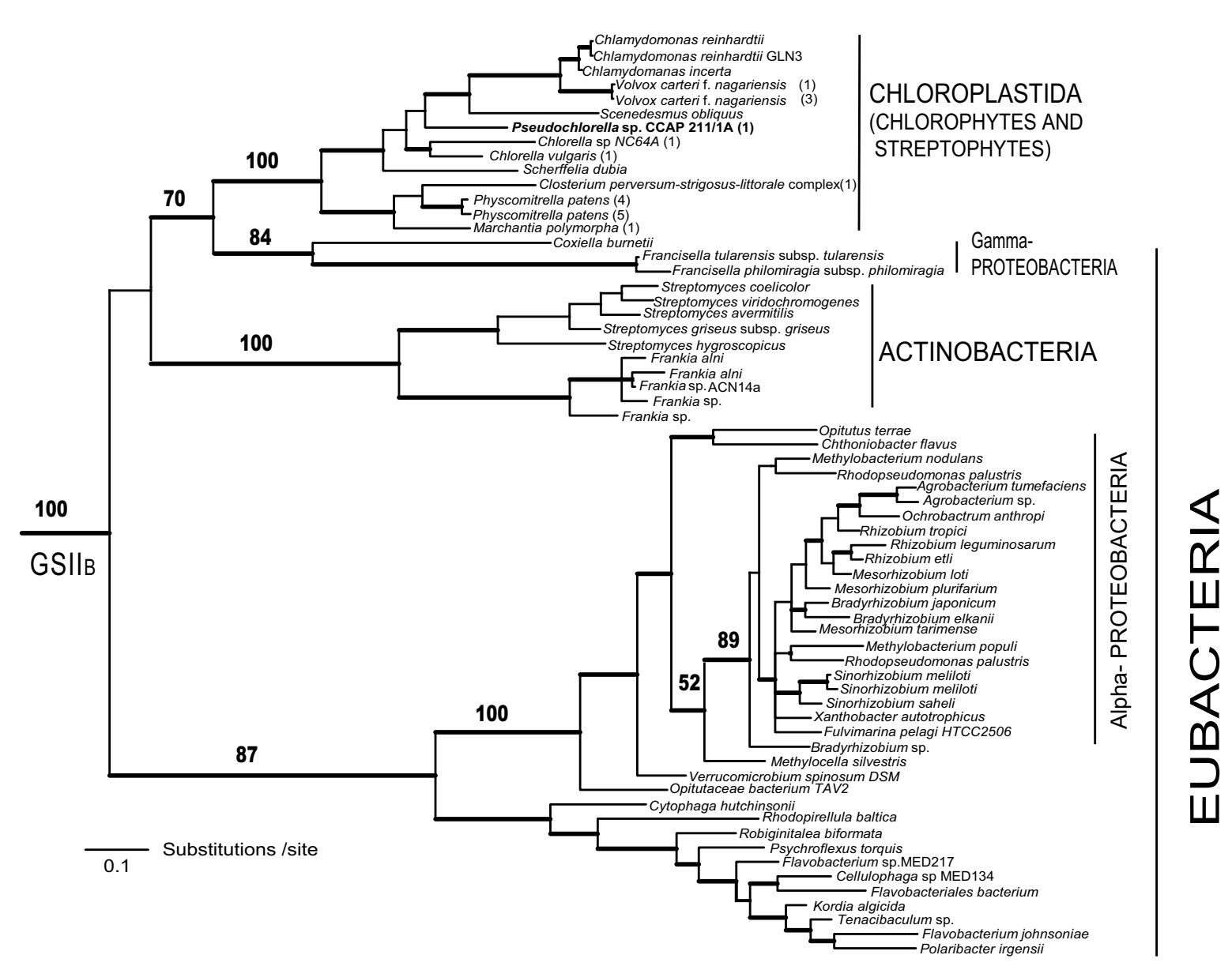

Figure 3 Evolutionary relationship of $\mathrm{GS} \|_{\mathrm{B}}$ genes from prokaryotes and eukaryotes, terminal taxa expanded. The inclusion of GSII ${ }_{B}$ genes from eukaryotes (early-diverging Chloroplastida) within prokaryotic $\mathrm{GSI}_{B}$ Clade is evidence of HGT from prokaryotes to eukaryotes within this group. Nodes with BBP support $>0.95$ are represented by thick lines. RAxML bootstrap values are indicated for major nodes. RAxML values are not indicated for terminal bifurcations. The sequence characterized in the present study is shown in bold.

\section{Estimating the timing of the HGT}

To estimate the relative and absolute timing of the HGT of $\mathrm{GSII}_{\mathrm{B}}$, we used Bayesian relaxed molecular clock analyses [28]. Both the uncalibrated (Figure 4) and calibrated analyses (Additional file 3) show an overlap of the 95\% highest density posterior node ranges of the origin of $\mathrm{GSII}_{\mathrm{B}}$ in the early-diverging Chloroplastida coinciding with the GSII $\mathrm{E}_{\mathrm{E}}$ divergence in the opisthokonts and in the primary photosynthetic eukaryotes (Archaeplastida). Our analyses indicate that $\mathrm{GSII}_{\mathrm{B}}$ and $\mathrm{GSII}_{\mathrm{E}}$ may have coexisted for an extended period of time and under this scenario, the putative timing of the HGT event from eubacteria to eukaryotes could be placed either prior to or after the divergence of the primary photosynthetic lineages. At present, there is no evidence of $\mathrm{GSII}_{B}$ in genomes of red algae (Cyanidioschyzon merolae [29] and
Galdieria sulphuraria [30,31]), or the glaucophyte Cyanophora paradoxa. We acknowledge that taxon sampling is not extensive within these two lineages and hence cannot exclude the possibility of the existence $\mathrm{GSII}_{\mathrm{B}}$ in these groups. However, given these limited data it is most parsimonious to assume that $\mathrm{GSII}_{\mathrm{B}}$ was acquired only by the Chloroplastida, early after the divergence from the Glaucophyta and Rhodophyceae (red algae).

The distribution of $\mathrm{GSII}_{\mathrm{B}}$ within Chloroplastida covers the major lineages of Chlorophyta (Chlorophyceae, Trebouxiophyceae, and Prasinophyceae; Additional file 1). In addition, a partial $\mathrm{GSII}_{B}$ sequence was identified in a member of the Ulvophyceae (Acetabularia acetabulum; (Additional files 2 and 4). Within Streptophyta, GSII $_{B}$ genes are present in Mesostigmatophyceae (Mesostigma viride; Additional files 2 and 4), Zygnemophyceae 
(Desmidiales; Closterium peracerosum-strigosum-littorale complex), Marchantiophyta (liverworts; Marchantia polymorpha) and Bryophyta (mosses; Physcomitrella patens). GSII $_{\mathrm{B}}$ is absent from the single Lycopodiophyta genome (Selaginella moellendorffii) and from all seed plants. Hence, we propose that $\mathrm{GSII}_{\mathrm{B}}$ was lost in the plant lineage after the colonization of land by early plants, marked by the divergence of bryophytes and lycopodiophytes, which is one of the oldest vascular plant lineages [32].

\section{Functional localization and $\mathrm{GSII}_{\mathrm{B}}$ gene duplication}

The Chloroplastida lineages that contain the $\mathrm{GSII}_{\mathrm{B}}$ gene also have a $\mathrm{GSII}_{\mathrm{E}}$ counterpart, which attaches to a basal node within the photosynthetic eukaryotes (Figure 1). Both the $\mathrm{GSII}_{\mathrm{B}}$ and $\mathrm{GSII}_{\mathrm{E}}$ genes are nuclear encoded and thus we identified the cellular location of each of the gene products based on the presence (organelle-localized) or absence (cytosol-localized) of $\mathrm{N}$-terminal transit peptides using TargetP ver. 1.1 ([33], see Additional file 5). None of the early-diverging Chloroplastida $\mathrm{GSII}_{\mathrm{E}}$ enzymes contained transit peptides. In contrast, chloroplast transit sequences were identified in the $\mathrm{GSII}_{\mathrm{B}}$ protein sequences from Chlorella sp. NC64A, C. vulgaris and the streptophyte, Closterium peracerosum-strigosum-littorale (Zygnemophyceae) but not in the moss (P. patens) or liverwort (M. polymorpha). Mitochondrial-targeting transit peptides were predicted in $\mathrm{GSII}_{\mathrm{B}}$ sequences from C. reinhardtii, Volvox carteri f. nagariensis and Scenedesmus obliquus (see Additional file 5). Previous work indicated that chloroplast transit sequences from $C$. reinhardtii shared features with both mitochondrial and higher plant chloroplast pre-sequences [34] and thus the prediction of a mitochondrial location of $\mathrm{GSII}_{\mathrm{B}}$ may not reflect its true functional localization. Alternatively, $\mathrm{GSII}_{\mathrm{B}}$ may be targeted to both the mitochondria and chloroplast, similar to what is observed for $\mathrm{GSII}_{\mathrm{E}}$ in leaves of some vascular plants $[35,36]$. While experimental evidence is required to confirm the cellular localization of the $\mathrm{GSII}_{\mathrm{B}}$, it appears that the $\mathrm{GSII}_{\mathrm{B}}$ enzymes function in either the chloroplast or mitochondrion in the chlorophytes and early-diverging streptophytes (Closterium sp.) and that GSII $_{E}$ functions in the cytosol.

The $\mathrm{GSII}_{\mathrm{B}}$ gene is duplicated in C. reinhardtii, V. carteri f nagariensis and $P$. patens. The duplicated copies of $\mathrm{GSII}_{\mathrm{B}}$ in C. reinhardtii and $V$. carteri $\mathrm{f}$ nagariensis were nearly identical ( $90 \%$ and $95 \%$ identical, respectively) and present in the genome in a head-to-head orientation. Similarly, the $\mathrm{GSII}_{\mathrm{B}}$ genes in P. patens were $98 \%$ identical but do not appear to be in close genomic proximity.
Within our phylogenetic analyses (Figure 3), the duplicated $\mathrm{GSII}_{\mathrm{B}}$ of $C$. reinhardtii, V. carteri f. nagariensis and $P$. patens each formed separate clades, suggesting the genes evolved by independent duplication events. Alternatively, the $\mathrm{GSII}_{\mathrm{B}}$ genes in $C$. reinhardtii and $V$. carteri may have evolved via an early duplication within the Chlamydomonadales with subsequent gene conversion following the divergence of these lineages. The $\mathrm{GSII}_{\mathrm{B}}$ are differentially expressed in $C$. reinhardtii suggesting the need for maintenance of both the copies in the organism [37].

\section{$\mathrm{GSII}_{B}$ loss and replacement of function}

In contrast to the expression of $\mathrm{GSII}_{\mathrm{E}}$ and $\mathrm{GSII}_{\mathrm{B}}$ genes in the early-diverging Chloroplastida, the chloroplast- and cytosolic-localized GSII enzymes in angiosperms are both members of the GSII family and form two distinct clades in our phylogenetic analyses (Figures 1 and 2). As predicted in earlier studies [19], the genes encoding these enzymes arose via a recent gene duplication event with further expansion in the number of genes encoding cytosolic isoenzymes in several plant lineages (Figure 2, $[38,39])$. Since $\mathrm{GSII}_{\mathrm{B}}$ is absent from vascular plants, it appears that the chloroplast function of $\mathrm{GSII}_{B}$ has been replaced by a gene duplication event in higher plants allowing for subsequent loss of the gene from this lineage. There is also an expansion of the $\mathrm{GSII}_{\mathrm{E}}$ gene family in gymnosperms (Figure 2), but the enzymes are all localized to the cytosol and the plastid targeted isoform appears to have been lost from this group. The expansion of the $\mathrm{GSII}_{\mathrm{E}}$ gene family coincides with the development of vascularization of land plants and maybe correlated with the partitioning of nitrogen assimilation between below and above ground tissue (see Additional file 3).

\section{Conclusions}

We have provided evidence of an ancient HGT event involving the gene for an essential enzyme, GSII. GSII has been well characterized at the molecular level in angiosperms but has been largely overlooked in the earlydiverging plant lineages, which were addressed in the present study. Although recent comparative genomic analyses failed to identify bacterial genes in Chlamydomonas reinhardtii [40], our discovery of a eubacteriallike GSII in the chlorophytes and early-diverging streptophytes suggests that further exploration within these lineages is merited. The branching pattern within the monophyletic assemblage of the chlorophytes and earlydiverging streptophytes is similar to other molecular and organismal phylogenies, suggesting the occurrence of a single HGT event. As a result, $\mathrm{GSII}_{\mathrm{B}}$ may be useful in 


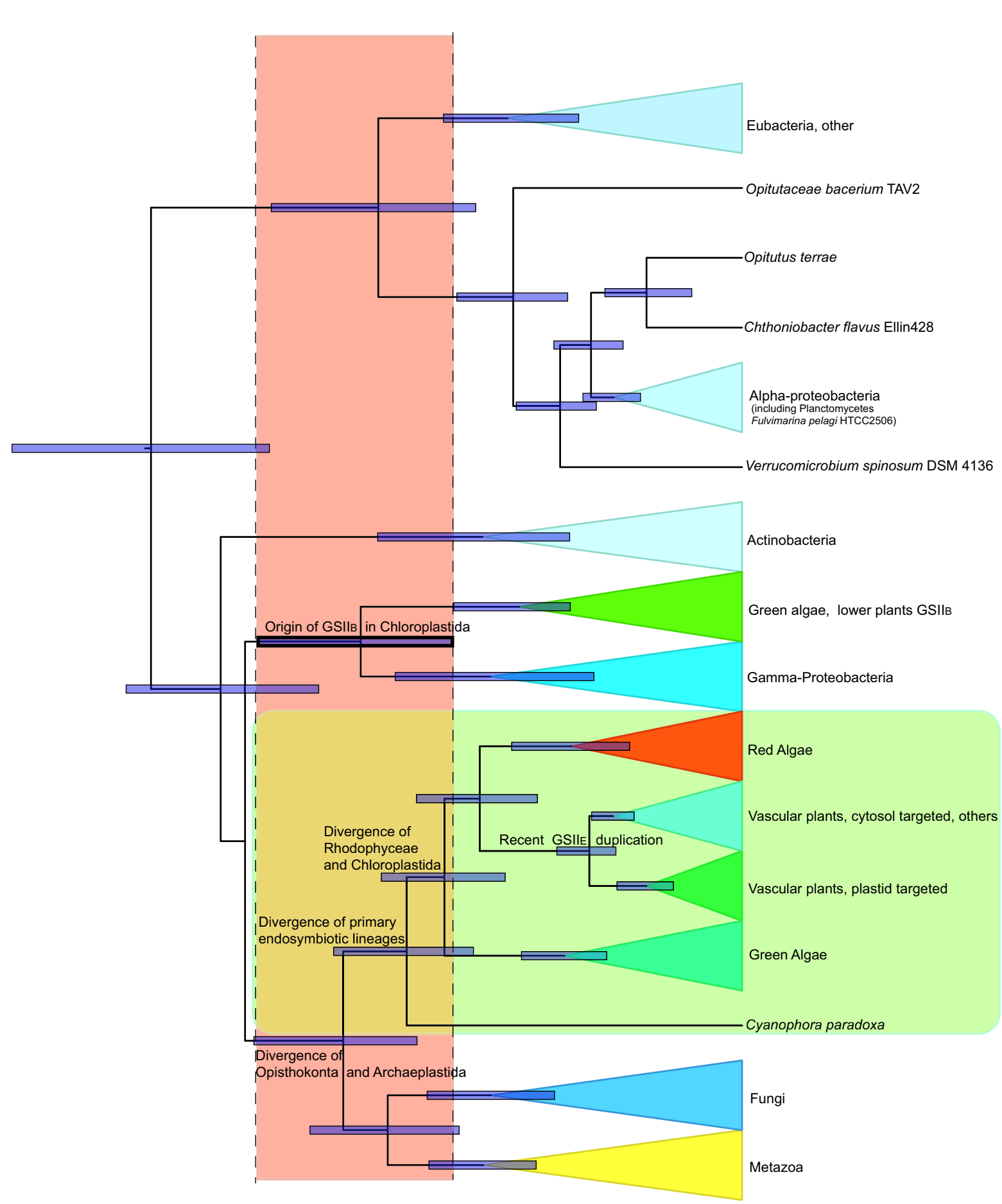

Figure 4 Maximum clade probability chronogram from the BEAST analysis of the $\mathrm{GSII}_{B}$ and $\mathrm{GSII}_{\mathrm{E}}$ amino acid sequence alignment. All lineages were allowed to evolve according to a relaxed molecular clock and WAG + Inv + Gamma model. Bars on nodes indicate the width of the $95 \%$ highest posterior density for each divergence time. 
resolving taxonomic associations within and among green algal and early-diverging streptophyte lineages.

Several genes of bacterial origin have been identified in Dictyostelium discoideum and are thought to be advantageous to organisms living in soil [40]. More recently, Richards et al. [41] identified five genes in plants that appear to be of fungal origin and argue that two may have been advantageous for organisms colonizing a terrestrial environment. We propose that the acquisition of enzymes by HGT results in a more rapid change in enzymatic capacity or kinetic diversity than evolution of isoenzymes by gene duplication and subsequent specialization. Biochemical studies have suggested that $\mathrm{GSII}_{\mathrm{B}}$ has a lower affinity for $\mathrm{NH}_{4}{ }^{+}$and Glu than $\mathrm{GSII}_{\mathrm{E}}$ [42], characteristics that would be advantageous for enzymes assimilating higher concentrations of $\mathrm{NH}_{4}{ }^{+}$from environmental sources, $\mathrm{NO}_{3}$-assimilation, or increased rates of photorespiration. Increased taxon sampling and an enlarged fossil age constraint dataset will allow for a more detailed examination of the timing of GSII gains and losses over geological history and coupled with major transitions in plant evolution.

\section{Methods}

\section{Algal cultures and sequencing}

Four members in the class Trebouxiophyceae were selected for GSII gene amplification. Cultures of Pseudochlorella sp. CCAP211/1A, Chlorella luteoviridis, and Auxenochlorella protothecoides were a gift from Dr. Peggy Winter (University of West Florida), and Prototheca zopfii was a gift from Dr. Drion Boucias (University of Florida). Cultures were grown axenically in ATCC medium 847, (Pseudochlorella sp. CCAP211/1A, C. luteoviridis and A. protothecoides) and in ATCC medium 28: Emmons' modification of Sabouraud's agar (P. zopfii) at $17^{\circ} \mathrm{C}$ and 12:12 h light: dark cycle. Cells were collected by centrifugation (approximately $50 \mathrm{~mL}$ of culture), flash frozen in liquid nitrogen, ground in a mortar and pestle and subjected to DNA and RNA extraction. DNA was extracted using a hexadecyltrimethylammonium bromide extraction protocol [43]. RNA was extracted using an RNeasy Mini Kit (Qiagen Inc., Valencia, CA) with modifications outlined in Brown et al. [44] for extraction with glass beads using bead beating. Extracted nucleic acids were quantified spectrophotometrically for downstream applications using a MWG BIOTECH Lambda Scan 200×, 96-well Microplate Reader with KCJunior Software (MWG BIOTECH, High Point, NC). cDNA was synthesized using an Omniscript RT kit (Qiagen Inc., Valencia, CA). Total RNA $(1.5 \mu \mathrm{g})$ was used as a template and the oligo-d (T) primer GCGGCCGCTCTAGACTAG(T) ${ }_{18}$ as the first strand primer. Primers were designed to target specifically $\mathrm{GSII}_{\mathrm{E}}$ and $\mathrm{GSII}_{\mathrm{B}}$ sequences. $\mathrm{GSII}_{\mathrm{E}}$ primers were based on existing sequences from vascular plants, chlorophytes and rhodophytes. $\mathrm{GSII}_{\mathrm{B}}$ primers were based on existing sequences from Chlamydomonas reinhardtii and Physcomitrella patens. PCR was performed in a final volume of $25 \mu \mathrm{L}$ with Taq PCR core kit (Qiagen) with Q solution to overcome problems associated with high GC content. Primer sequences are listed in Table 2. Thermal conditions for $\mathrm{GSII}_{\mathrm{E}}$ : 30 cycles of $95^{\circ} \mathrm{C}$ for 30 s, $50^{\circ} \mathrm{C}$ for $30 \mathrm{~s}, 72^{\circ} \mathrm{C}$ for $1 \mathrm{~min}$, performed for 30 cycles. Thermal conditions for $\mathrm{GSII}_{\mathrm{B}}$ : Initial denaturation of $94^{\circ} \mathrm{C}$ for 2 min, followed by 35 cycles of $94^{\circ} \mathrm{C}$ for $1 \mathrm{~min}, 51^{\circ} \mathrm{C}$ for 1 min, $72^{\circ} \mathrm{C}$ for $1 \mathrm{~min}$ and extension at $72^{\circ} \mathrm{C}$ for $5 \mathrm{~min}$.

Nested PCR amplification was used to obtain $\mathrm{GSII}_{B}$ sequences with first round done with cDNA and primer concentrations of $0.4 \mu \mathrm{M}$ (MossGS2-1F [forward] and MossGS2-2R [reverse]). The amplicon was used for a second round of amplification with primers Green UNI 1-F (forward) and cpGSII(QGPFY)-R (reverse) and yielded a DNA fragment of $330 \mathrm{bp}$. Amplified sequences were cleaned and sequenced either commercially (MWG Biotech, Charlotte, NC and Macrogen, Seoul, South Korea) or at Clark University using an automated DNA sequencer (ABI 3130), with ABI Prism Terminator Big Dye ver 3.1 (Applied Biosystems, Carlsbad, CA). Some PCR products were cloned into TOPO vectors following the manufacturer's protocol (TOPO TA Cloning Kit for Sequencing, Invitrogen, Carlsbad, CA) prior to sequencing. Rapid Amplification of cDNA Ends (RACE) methods were used to obtain the entire open reading frame for GSII $_{\mathrm{E}}$ sequences from Pseudochlorella sp. CCAP211/1A, C. luteoviridis, A. protothecoides and P. zopfii and partial $\mathrm{GSII}_{\mathrm{B}}$ sequence from Pseudochlorella sp. CCAP211/1A. 3' RACE reactions used a combination of gene specific primers and a portion of the oligo-d (T) primer (GCGGCCGCTCTAGACTAGT) used for cDNA synthesis. 5' RACE reactions were performed using 5' RACE System version 2.0 from Invitrogen (Invitrogen) and SMART ${ }^{\mathrm{m}}$ RACE cDNA Amplification Kit (Clontech Laboratories Inc., Mountain View, CA) following manufacturers' recommendations. Contigs were assembled using CodonCode Aligner (CodonCode Corporation, Deadham, MA). All sequences were translated into amino acids in silico.

\section{Phylogenetic analyses}

GSII sequences were retrieved from public databases as well as genome and EST projects using the GSII sequence from the diatom Skeletonema costatum (AAC77446) as query, or glutamine synthetase as a keyword. Subsequent queries with eubacterial GSII sequences did not retrieve any additional sequences. Complete information on taxa, database sources and accession numbers is provided in 
Table 2: Primers used for amplification of GSII genes from green algae

\begin{tabular}{|c|c|c|c|}
\hline Gene & Primer name & Direction & Sequence \\
\hline & MossGS2-1F & Forward & 5'-TGGGTTGATGGTMANGARGG-3' \\
\hline \multirow[t]{4}{*}{$\mathrm{GSII}_{\mathrm{B}}$} & MossGS2-2R & Reverse & 5'-ATNCCGAAMTCTTCNCC-3' \\
\hline & Green UNI 1-F & Forward & 5'-CCIRAITGGWSITTYGAYGG-3' \\
\hline & cpGSII(QGPFY)-R & Reverse & 5'-CCRCARTARAAIGGICCYTGIGG-3' \\
\hline & GALG GS F & Forward & $5^{\prime}$ - TGC CCA TCC CCA CCA ACA C - 3' \\
\hline \multirow[t]{3}{*}{$\mathrm{GSI}_{\mathrm{E}}$} & GALG GS R & Reverse & 5' - TCT CGT GCT TGC CCG TCA GG - 3' \\
\hline & GS2ChloroF & Forward & 5' - CGG CWT CGA GCA GGA GTA CAC - 3' \\
\hline & GS2ChloroR & Reverse & 5' - CCG AYC TGG WAC TCC CAC TGG - 3' \\
\hline
\end{tabular}

Sequences of degenerate primers are presented using IUBMB single letter codes. I represents inosine.

Additional file 1. The initial alignment of amino acid sequences was done with the web based program CLUSTAL W, using default parameters [45], followed by manual adjustment using BioEdit Sequence Alignment Editor [46] and MacClade 4.08 [47]. The N- and C terminal ends of the proteins along with highly variable regions within the alignments were excluded in the phylogenetic analyses.

The final GSII alignment consisted of 196 taxa and 333 characters for Bayesian analysis. Trees were inferred by calculating Bayesian posterior probabilities using MrBayes 3.1.2 [48,49]. Two parallel runs, each with four chains (three heated and one cold) were run for $10^{6}$ generations. The evolutionary models implemented in MrBayes3.1.2 were explored using the mixed amino acid model. Rate variation across sites was approximated using a gamma distribution with proportion of invariable sites estimated from the data. Trees were sampled every 100 generations. Likelihood tree scores of two independent runs were plotted to estimate the point of convergence to a stable likelihood, and to determine the trees to be excluded via "burnin." Bayesian posterior probabilities of the branches were calculated from trees from both the runs, totaling 20,002 trees. Trees remaining $(10,000)$ after a burnin of 5001 for each run were used to compute a $50 \%$ majority-rule consensus.

Maximum likelihood (ML) based inference of the phylogenetic trees was done using the software RAxML 7.0.4 $[50,51]$. The analysis used a random starting tree and the rapid hill-climbing algorithm (i.e., option - $\mathrm{f} d$ in RAxML) and the WAG model of amino acid substitution were used. A random seed number was used to turn on rapid bootstrapping (-x) and 1000 bootstrap trees were generated by invoking -\# 1000 and - x options in RAxML. A majority rule consensus tree was created in PAUP* $4.0 \mathrm{~b}$ [52]. The phylogenetic trees in figures 1, 2 \&3 are the $50 \%$ majority rule consensus trees from the Bayesian analyses on which the RAxML bootstrap values have been indicated. The eubacterial GSII $_{B}$ sequences were used as the monophyletic outgroup in the graphical representation of the phylogenies.

\section{Prediction of functional localization of $\mathrm{GSII}_{\mathrm{B}}$ and $\mathrm{GSII} \mathrm{E}_{\mathrm{E}}$ protein sequences in early-diverging Chloroplastida}

We used the web-based programs TargetP 1.1[33] and ChloroP 1.1 [53] to identify N-terminal transit peptides in $\mathrm{GSII}_{\mathrm{B}}$ and $\mathrm{GSII}_{\mathrm{E}}$ proteins (see Additional file 5).

\section{Estimation of divergence times}

We estimated the divergence times using Bayesian approach implemented in BEAST 1.4.8 [28]. We did an un-calibrated and calibrated run. A relaxed molecular clock model of uncorrelated log normal distribution was used. For the un-calibrated analysis, a starting tree generated by RAxML 7.0.4 [50] was used as the input tree with the GS amino acid sequence alignment. For the calibrated analysis we set uniform priors on tmrca parameter. Fossil dates were used as minimum dates and were, as follows, Ascomycota, 400 MYA [54], Bilateria, 550 MYA [55] and streptophytes 475 MYA [56]. Secondary age constraints based on published estimates of divergence times were not used. We used the following models, WAG + Inv + Gamma with priors, birth death speciation on the tree. Markov Chain Monte Carlo was set to default 10 million with sampling at every 1000 generation, resulting in 10,000 trees. Convergence was assessed in Tracer v 1.4 [57] and the first three million samples were excluded as burnin. A maximum clade credibility tree was generated by analyzing the BEAST tree file in TreeAnnotator 1.4.6 [58]. This program determined the $95 \%$ highest posterior densities and estimated the node heights as mean heights. 


\section{Additional material}

Additional file $1 \mathrm{GSII}$ protein sequences used in the present study GenBank accession numbers and JGI DOE scaffold and protein ID information for the GSII proteins are provided.

Additional file 2 Identification of $\mathrm{GSII}_{\mathrm{B}}$ sequences from Acetabularia acetabulum and Mesostigma viride. The data matrix used in this analyses is described in the Methods with sequences from the following taxa included in the alignment: Acetabularia acetabulum (Chlorophyta, Ulvophy ceae), Mesostigma viride (Streptophyta, Mesostigmatophyceae), which are shown in bold. Pseudochlorella sp. CCAP211/1A (1) GSII amplified in the present study is also shown in bold. Tree used for this illustration is a representative derived from parsimony heuristic search analysis. RAxML bootstrap values are shown for the major clades of GSII genes, which were derived from 1000 bootstrap replicates with the parameters described in the Methods.

Additional file 3 Maximum clade probability tree displayed as a chronogram from the BEAST analysis of the GS amino acid sequence alignment. All lineages evolved according to a relaxed molecular clock and WAG + Inv. + Gamma model. Node bars indicate the width of the $95 \%$ highest posterior density with minimum and maximum values in parentheses. Bold numbers near the nodes indicate node ages. Major lineages are depicted as collapsed triangles. Nodes for which fossil dates were used are marked as A $=$ Acsomycota, 400MYA, B = Bilateria 550MYA and C $=$ Streptophytes 475 MYA. $P=$ Paleozoic; $M=$ Mesozoic; $C=$ Cenozoic; $C a m b .=$ Cambrian.

Additional file 4 Partial GSII ${ }_{B}$ sequences were identified in two additional Chloroplastida by preliminary phylogenetic analyses. Partial $\mathrm{GSII}_{\mathrm{B}}$ sequences were obtained for Acetabularia acetabulum (Chlorophyta: Ulvophyceae) and Mesostigma viride (Streptophyta: Mesostigmatophyceae). These sequences were not included in the phylogenetic analyses presented within the paper (figures 1, 2, 3 and 4) due to their short length. Phylogenetic analysis confirming these proteins as members of the GSII ${ }_{B}$ clade is presented in Additional file 2 .

\section{Additional file 5 Predicted cellular localization of GSII proteins in} early-diverging Chloroplastida. Only taxa containing both $\mathrm{GSI}_{\mathrm{B}}$ and $\mathrm{GSI} \mathrm{E}_{\mathrm{E}}$ within Chloroplastida were analyzed. N-terminal sequences of GSII proteins were analyzed for organellar transit peptides, in silico. References are given for established functional localizations.

\section{Abbreviations}

GS: Glutamine synthetase; HGT: Horizontal gene transfer; BPP: Bayesian posterior probability;

\section{Authors' contributions}

SG participated in the cloning and sequencing of GSII genes, assembled the data matrix, conducted all phylogenetic analyses, and drafted the manuscript. MB participated in the execution and interpretation of the relaxed molecular clock analyses and assisted in the writing of the manuscript. AT was responsible for the cloning and sequencing of GSII genes and assisted in writing the manuscript. DLR conceived of the study, coordinated the research, and helped draft the manuscript. All authors read and approved the final manuscript.

\section{Acknowledgements}

We thank Jenna Nguyen and Jacqueline Mitchell for their assistance with this project and David Hibbett for helpful discussions regarding the phylogenetic analyses. We also thank two anonymous reviewers and the editor for their constructive comments and recommendations. This research was supported by an NSF CAREER Award (IBN 0238426) to DLR.

\section{Author Details}

${ }^{1}$ Clark University, Biology Department, 950, Main Street, Worcester, MA 01610 USA and ${ }^{2}$ Nova Southeastern University, 3301 College Avenue, Fort Lauderdale, FL 33314, USA

Received: 23 December 2009 Accepted: 25 June 2010 Published: 25 June 2010

\section{References}

1. Kumada Y, Benson DR, Hillemann D, Hosted TJ, Rochefort DA, Thompson CJ, Wohlleben W, Tateno Y: Evolution of the glutamine synthetase gene, one of the oldest existing and functioning genes. Proc Natl Acad Sci USA 1993, 90:3009-3013.

2. Pesole G, Bozzetti MP, Lanave C, Preparata G, Saccone C: Glutamine synthetase gene evolution: A good molecular clock. Proc Natl Acad Sci USA 1991, 88:522-526.

3. Brown JR, Masuchi Y, Robb FT, Doolittle WF: Evolutionary relationships of bacterial and archaeal glutamine synthetase genes. J Mol Evol 1994 38:566-576

4. Eisenberg D, Gill HS, Pfluegl GMU, Rotstein SH: Review: Structurefunction relationships of glutamine synthetases. Biochim Biophys Acta 2000, 1477:122-145.

5. Garcia-Dominguez M, Reyes JC, Florencio FJ: Purification and characterization of a new type of glutamine synthetase from cyanobacteria. Eur J Biochemistry 1997, 244:258-264

6. Reyes JC, Florencio FJ: A new type of glutamine synthetase in cyanobacteria: The protein encoded by the $g \ln N$ gene supports nitrogen assimilation in Synechocystis sp. strain PCC 6803. J Bacter 1994, 176:1260-1267.

7. Robertson DL, Alberte RS: Isolation and characterization of glutamine synthetase from the marine diatom Skeletonema costatum. Plant Physiol 1996, 111:1169-1175.

8. Robertson DL, Smith GJ, Alberte RS: Glutamine synthetase in marine algae: new surprises from an old enzyme. J Phycol 2001, 37:793-795.

9. Brown JR, Doolittle WF: Archaea and the prokaryote-to-eukaryote transition. Microbiol Mol Biol Rev 1997, 61:456-502.

10. Mathis R, Gamas P, Meyer Y, Cullimore JV: The presence of GSI-like genes in higher plants: Support for the paralogous evolution of GSI and GSII. J Mol Evol 2000, 50:116-122.

11. Robertson DL, Tartar A: Evolution of glutamine synthetase in heterokonts: evidence for endosymbiotic gene transfer and the early evolution of photosynthesis. Mol Biol Evol 2006, 23:1048-1055.

12. Carlson TA, Chelm BK: Apparent eucaryotic origin of glutamine synthetase II from the bacterium Bradyrhizobium japonicum. Nature 1986:568-570.

13. Shatters RG, Kahn ML: Glutamine synthetase II in Rhizobium reexamination of the proposed horizontal transfer of DNA from eukaryotes to prokaryotes. J Mol Evol 1989, 29:422-428.

14. Adl SM, Simpson AGB, Farmer MA, Andersen RA, Anderson OR, Barta JR, Bowser SS, Brugerolle G, Fensome RA, Frederica S, James TY, Karpov S, Kugrens P, Krug J, Lane CE, Lewis LA, Lodge J, Lynn DH, Mann DG, Mccourt RM, Mendoza L, Moestrup $\varnothing$, Mozley-Standridge SE, Nerad TA, Shearer CA, Smirnov AV, Spiegel FW, Taylor MFJR: The new higher level classification of eukaryotes with emphasis on the taxonomy of protists. J Eukaryot Microbiol 2005, 5:399-451

15. Coruzzi GM: Primary N-assimilation into Amino Acids in Arabidopsis. In The Arabidopsis Book Edited by: Somerville C, Meyerowitz E. Rockville, MD: American Society of Plant Biologists; 2003:1-17.

16. Lam H-M, Coschigano KT, Oliveira IC, Melo-Oliveira R, Coruzzi GM: The molecular-genetics of nitrogen assimilation into amino acids in higher plants. Annu Rev Plant Physiol Mol Biol 1996, 47:569-593.

17. Sechley KA, Yamaya T, Oaks A: Compartmentation of nitrogen assimilation in higher plants. Int Rev Cytol 1992, 134:85-163.

18. Woodall J, Boxall JG, Forde BG, Pearson J: Changing perspectives in plant nitrogen metabolism: the central role of glutamine synthetase. Science Progress 1996, 79:1-26.

19. Coruzzi GM, Edwards JW, Tingey SV, Tsai F-Y, Walker EL: Glutamine synthetase: molecular evolution of an eclectic multi-gene family. In The Molecular Basis of Plant Development. Proceedings of an E.I. duPont Nermous-UCLA Symposium Edited by: Goldberg R. New York: Alan R. Liss, Inc; 1989:223-232

20. Robertson DL, Smith GJ, Alberte RS: Characterization of a cDNA encoding glutamine synthetase from the marine diatom Skeletonema costatum (Bacillariophyceae). J Phycol 1999, 35:786-797.

21. Ahmad I, Hellebust JA: Nitrogen metabolism of the marine microalga Chlorella autotrophica. Plant Physiol 1984, 76:658-663.

22. Casselton PJ, Chandler G, Shah N, Stewart GR, Sumar N: Glutamine synthetase isoforms in algae. New Phytol 1986, 102:261-270. 
23. Chen $Q$, Silflow CD: Isolation and characterization of glutamine synthetase genes in Chlamydomonas reinhardtii. Plant Physiol 1996, 112:987-996

24. Suarez MF, Avila C, Gallardo F, Canton FR, Garcia-Gutierrez A, Claros MG, Canovas FM: Molecular and enzymatic analysis of ammonium assimilation in woody plants. J Exp Bot 2002, 53:891-904

25. Bowman JL, Floyd SK, Sakakibara K: Green genes - comparative genomics of the green branch of life. Cell 2007, 129:229-234.

26. Dufresne A, Salanoubat M, Partensky Fdr, Artiguenave Fo, Axmann IM, Barbe Vr, Duprat S, Galperin MY, Koonin EV, Le Gall F, Makarova KS, Ostrowski M, Oztas S, Robert C, Rogozin IB, Scanlan DJ, de Marsac NT, Weissenbach J, Wincker P, Wolf YI, Hess WR: Genome sequence of the cyanobacterium Prochlorococcus marinus SS120, a nearly minimal oxyphototrophic genome. Proc Natl Acad Sci USA 2003, 100:10020-10025.

27. Palenik B, Brahamsha B, Larimer FW, Land M, Hauser L, Chain P, Lamerdin J, Regala W, Allen EE, McCarren J, Paulsen I, Dufresne A, Partensky F, Webb EA, Waterbury J: The genome of a motile marine Synechococcus. Nature 2003, 424:1037-1042.

28. Drummond AJ, Rambaut A: BEAST: Bayesian evolutionary analysis by sampling trees. BMCEvol Biol 2007, 7:214.

29. Matsuzaki M, Misumi O, Shin-i T, Maruyama S, Takahara M, Miyagishima Sy, Mori T, Nishida K, Yagisawa F, Nishida K, Yoshida Y, Nishimura Y, Nakao S, Kobayashi T, Momoyama Y, Higashiyama T, Minoda A, Sano M, Nomoto H, Oishi K, Hayashi H, Ohta F, Nishizaka S, Haga S, Miura S, Morishita T, Kabeya Y, Terasawa K, Suzuki Y, Ishii Y, et al:: Genome sequence of the ultrasmall unicellular red alga Cyanidioschyzon merolae 10D. Nature 2004, 428:653-657.

30. Barbier G, Oesterhelt C, Larson MD, Halgren RG, Wilkerson C, Garavito RM, Benning C, Weber APM: Comparative genomics of two closely related unicellular thermo-acidophilic red algae, Galdieria sulphuraria and Cyanidioschyzon merolae, reveals the molecular basis of the metabolic flexibility of Galdieria sulphuraria and significant differences in carbohydrate metabolism of both algae. Plant Physiol 2005, 137:460-474.

31. Weber A, Oesterhelt C, Gross W, Bräutigam A, Imboden L, Krassovskaya I, Linka N, Truchina J, Schneidereit J, Voll H, Voll L, Zimmermann M, Jamai A, Riekhof W, Yu B, Garavito R, Benning C: EST-analysis of the thermoacidophilic red microalga Galdieria sulphuraria reveals potential for lipid $A$ biosynthesis and unveils the pathway of carbon export from rhodoplasts. Plant Mol Biol 2004, 55:17-32.

32. Weng J-K, Tanurdzic M, Chapple C: Functional analysis and comparative genomics of expressed sequence tags from the lycophyte Selaginella moellendorffii. BMC Genomics 2005, 6:85

33. Emanuelsson O, Nielsen H, Brunak S, von Heijne G: Predicting subcellular localization of proteins based on their $\mathrm{N}$-terminal amino acid sequence. J Mol Biol 2000, 300:1005-1016.

34. Franzén LG, Rochaix JD, von Heijne G: Chloroplast transit peptides from the green alga Chlamydomonas reinhardtii share features with both mitochondrial and higher plant chloroplast presequences. FEBS Lett 1990, 260:165-168.

35. Taira M, Valtersson U, Burkhardt B, Ludwig RA: Arabidopsis thaliana GLN2encoded glutamine synthetase is dual targeted to leaf mitochondria and chloroplasts. Plant Cell 2004, 16:2048-2058.

36. Linka M, Weber APM: Shuffling ammonia between mitochondria and plastids during photorespiration. Trends Plant Sci 2005, 10:461-465.

37. Vallon O, Spalding M: Amino Acid Metabolism. In The Chlamydomonas Source Book Volume 2. 2nd edition. Edited by: Stern D, Harris EH. Boston: Elsevier; 2008:115-150

38. Doyle JJ, Doyle JL, Harbison C: Chloroplast-expressed glutamine synthetase in Glycine and related Leguminosae: Phylogeny, gene duplication, and ancient polyploidy. Systematic Botany 2003, 28:567-577

39. Walker EL, Weeden NF, Taylor CB, Green P, Coruzzi GM: Molecular evolution of duplicate copies of genes encoding cytosolic glutamine synthetase in Pisum sativum. Plant Mol Biol 1995, 29:1111-1125.

40. Keeling PJ, Palmer JD: Horizontal gene transfer in eukaryotic evolution. Nat Rev Genet 2008, 9:605-618.

41. Richards TA, Soanes DM, Foster PG, Leonard G, Thornton CR, Talbot NJ: Phylogenomic analysis demonstrates a pattern of rare and ancient horizontal gene transfer between plants and fungi. Plant Cell 2009, 21:1897-1911.
42. Florencio FJ, Vega JM: Separation, purification, and characterization of two isoforms of glutamine synthetase from Chlamydomonas reinhardii. ZNaturforsch 1983, 38c:531-538.

43. Coyer JA, Robertson DL, Alberte RS: Genetic variability within a population and between diploid/haploid tissue of Macrocystis pyrifera (Phaeophyceae). J Phycol 1994, 30:545-542.

44. Brown KL, Twing Kl, Robertson DL: Unraveling the regulation of nitrogen assimilation in the marine diatom Thalassiosira pseudonana (BACILLARIOPHYCEAE): Diurnal variations in transcript levels for five genes involved in nitrogen assimilation. J Phycol 2009, 45:413-426.

45. Thompson JD, Higgins DG, Gibson TJ: Clustal W: Improving the sensitivity of progressive multiple sequence alignment through sequence weighting, positions-specific gap penalties and weight matrix choice. Nucleic Acid Res 1994, 22:4673-4680

46. Hall TA: BioEdit: A user-friendly biological sequence alignment editor and analysis program for Windows 95/98/NT. Nucl Acids Symp Ser 1999, 41:95-98.

47. Maddison WP, Maddison DR: MacClade 4: Analysis of phylogeny and character evolution. Sunderland, MA: Sinauer Associates; 2000.

48. Huelsenbeck J, Ronquist F: MRBAYES: Bayesian inference of phylogeny Bioinformatics 2001, 17:754-755.

49. Ronquist F, Huelsenbeck JP: MRBAYES 3: Bayesian phylogenetic inference under mixed models. Bioinformatics 2003, 19:1572-1574.

50. Stamatakis A: RAxML-VI-HPC: Maximum Likelihood-based phylogenetic analyses with thousands of taxa and mixed models. Bioinformatics 2006, 22:2688-2690.

51. Stamatakis A, Hoover $P$, Rougemont J: A rapid bootstrap algorithm for the RAxML web servers. Syst Bio/ 2008, 57:758-771.

52. Swofford DL: PAUP: Phylogenetic analysis using parsimony, beta version 4.0b1. Sinauer Associates, Inc., Sunderland; 1998.

53. Emanuelsson $\mathrm{O}$, Nielsen $\mathrm{H}$, von Heijne $\mathrm{G}$ : ChloroP, a neural network based method for predicting chloroplast transit peptides and their cleavage sites. Prot Sci 1999, 8:978-984.

54. Taylor TN, Hass H, Herp H: The oldest fossil ascomycetes. Nature 1999, 399:648.

55. Valentine JW: The macroevolution of phyla. In Origin and Early Evolution of the Metazoa Edited by: Lipps JH, Signor PW. New York: Plenum Press; 1992:525-553.

56. Gray J: Major Paleozoic land plant evolutionary bio-events. Palaeogeogr Palaeoclimatol Palaeoecol 1993, 104:153-169.

57. Rambaut A, Drummond A: Tracer v1.4. 2007 [http://beast.bio.ed.ac.uk/ Tracer].

58. Rambaut A, Drummond AJ: TreeAnnotator 1.4.5. 2006 [http:// beast.bio.ed.ac.uk/TreeAnnotator].

doi: $10.1186 / 1471-2148-10-198$

Cite this article as: Ghoshroy et al., Molecular evolution of glutamine synthetase II: Phylogenetic evidence of a non-endosymbiotic gene transfer event early in plant evolution BMC Evolutionary Biology 2010, 10:198

\section{Submit your next manuscript to BioMed Central and take full advantage of:}

- Convenient online submission

- Thorough peer review

- No space constraints or color figure charges

- Immediate publication on acceptance

- Inclusion in PubMed, CAS, Scopus and Google Scholar

- Research which is freely available for redistribution 